\title{
The role of orbital order in the stabilization of the $(\pi, 0)$ ordered magnetic state in a minimal two-band model for iron pnictides
}

\author{
Sayandip Ghosh ${ }^{1, a)}$ and Avinash Singh ${ }^{1}$ \\ Department of Physics, Indian Institute of Technology Kanpur - 208016
}

Spin wave excitations and stability of the $(\pi, 0)$ ordered magnetic state are investigated in a minimal two-band itinerant-electron model for iron pnictides. Presence of hopping anisotropy generates a strong ferro-orbital order in the $d_{x z}$ and $d_{y z}$ Fe orbitals. The orbital order sign is as observed in experiments. The induced ferroorbital order strongly enhances the spin wave energy scale and stabilizes the magnetic state by optimizing the strength of the emergent AF and F spin couplings through optimal band fillings in the two orbitals. The calculated spin-wave dispersion is in quantitative agreement with neutron scattering measurements. Finite inter-orbital Hund's coupling is shown to further enhance the spin wave energies state by coupling the two magnetic sub-systems. A more realistic two-band model with less hopping anisotropy is also considered which yields not only the circular hole pockets, also correct ferro-orbital order and emergent $\mathrm{F}$ spin coupling.

PACS numbers: 75.25.Dk, 74.70.Xa, 75.30.Ds, 71.10.Fd

\section{INTRODUCTION}

The iron pnictides exhibit a typical phase diagram ${ }^{1,2}$ in which the parent compound goes through a tetragonalto-orthorhombic structural phase transition (at $T_{S}$ ) and spin ordering transition (at $T_{N}$ ). Upon doping, both transitions are suppressed and superconductivity emerges. Single crystal neutron scattering experiments show that Fe moments align antiferromagnetically (AF) along the $a$ direction and ferromagnetically $(\mathrm{F})$ along the $b$ direction ${ }^{3}$, so that the magnetically ordered state can be viewed as a $(\pi, 0)$ ordered spin density wave (SDW) state. Inelastic neutron scattering (INS) experiments $\underline{\underline{4}-6}$ yield well-defined spin-wave excitations up to the $\mathrm{F}$ zone boundary $\boldsymbol{q}=(0, \pi)$ on an energy scale $\sim 200 \mathrm{meV}$.

Several weak coupling models with Fermi surface (FS) nesting have been proposed ${ }^{7-9}$ to account for the observed magnetic order. Although these models can explain low-energy magnetic excitations, they fail at higher energies and suggest that spin waves enter the particle-hole continuum at high energies and become over-damped Stoner-type excitations ${ }^{9}$. The recently observed existence ${ }^{6}$ of spin-wave excitations even above $T_{N}$ is also contrary to this weak coupling nesting picture, within which there is no difference between moment melting and moment disordering temperatures. In fact, LDA calculations $\frac{10,11}{}$ suggest that $\mathrm{Fe}$ onsite interaction $U$ is comparable to Fe $3 d$ bandwidth (W) indicating that iron pnitides are moderately correlated materials.

Apart from the magnetic excitations, angle-resolved photoemission spectroscopy (ARPES) $12-14$ and X-ray linear dichroism (XLD) experiments 15 have clearly revealed the existence of orbital order in these materials. In the magnetic state, the Fe $d_{y z}$ band is shifted up relative to the $d_{x z}$ band ${ }^{13,15}$, causing electron density difference between the two orbitals. This type of orbital ordering

\footnotetext{
${ }^{a)}$ Electronic mail: sayandip@iitk.ac.in
}

was previously proposed ${ }^{16-19}$ to explain experimentally observed in-plane anisotropic behavior like anisotropy in magnetic exchange coupling ${ }^{4}$, transport properties 20,21 , FS structure ${ }^{13}$, and electronic structure 22 .

Is there a significant correlation between this observed orbital ordering and stability of the $(\pi, 0)$ magnetic state? In this paper, we will investigate the effect of orbital ordering on the SDW state stability within a minimal twoband itinerant-electron model. We will study how the variations in orbital disparity affects the induced $\mathrm{AF}$ and F spin couplings. This provides a microscopic understanding of the role of orbital ordering on the stability of the $(\pi, 0)$ ordered SDW state in the relevant intermediate coupling regime.

The minimal two-band model proposed earlier by Raghu et $a l^{23}$ gave Fermi surface structure consistent with LDA calculations at half-filling. Although nesting between hole and electron Fermi pockets yields low critical value of $U$ for $(\pi, 0)$ ordering, it has three major shortcomings: (i) no $\mathrm{F}$ spin coupling is generated due to nesting as shown by the vanishing spin wave energy at the $\mathrm{F}$ zone boundary 24,25 , whereas INS experiments yield maximum spin wave energy, (ii) no orbital ordering is obtained in this model for electron filling corresponding to nesting condition, and (iii) Fermi surface nesting is relevant only in weak coupling limit, whereas pnictides are in intermediate coupling regime $(U \sim W)$. At intermediate coupling, nesting is not very relevant for magnetic ordering as evidenced by the observation of a stable $(\pi, 0)$ state in one-band Hubbard model ${ }^{26}$.

Evidently, modifications are required to this two-band model and therefore investigation of models and mechanisms beyond nesting become relevant in order to reproduce experimentally observed orbital ordering as well as the spin wave dispersion. The present study is an investigation in this direction. As hopping anisotropy has been shown to yield orbital order in the $(\pi, 0) \operatorname{state}^{27}$, we will first consider the extreme hopping anisotropy case within a minimal two-band model (Section 2) and investigate the consequences on spin couplings and spin 
wave dispersion (Section 3), to bring out in a physically transparent manner how the resulting orbital order enhances the $\mathrm{F}$ and $\mathrm{AF}$ spin couplings in stabilizing the $(\pi, 0)$ structure and yielding the observed features of the spin wave dispersion. Guided by the above investigations, Section 4 describes modifications to the two-band model to obtain circular hole pockets and orbital order in agreement with experiments, and also emergence of required $\mathrm{F}$ spin coupling. This comprehensive approach of simultaneously keeping account of the Fermi surface as well as emergent spin couplings and spin wave dispersion provides important physical insight into further extension to a three-band model.

\section{MINIMAL TWO-BAND MODEL}

The iron pnictides have a quasi two-dimensional structure with layers of FeAs stacked along the $c$ axis. Among the five Fe $3 d$ orbitals, only $d_{x z}$ and $d_{y z}$ contribute to orbital ordering (due to $C_{4}$ symmetry of others) and we retain only these two in our model. The hybridization of Fe $3 d$ orbitals with themselves as well as through the As $3 p$ orbitals lying above and below the square plaquettes formed by the Fe atoms leads to hopping parameters in our two-orbital model. The hopping amplitudes are shown in Fig. 1 in which the $d_{x z}$ and $d_{y z}$ orbitals are taken to be extended along $x(a)$ and $y(b)$ direction respectively since the cores of $\mathrm{Fe} d_{x z}$ and $d_{y z}$ Wannier Functions extend towards the direction of magnetic ordering $\frac{17}{}$. Although hybridization between the orbitals can lead to finite $t_{\pi}$ (i.e. $\pi$ overlap), for simplicity we consider $t_{\sigma}$ (i.e. $\sigma$ overlap) only in our model. However, orbital order in our model persists as long as there is hopping anisotropy i.e. $t_{\sigma}$ is larger than $t_{\pi}$. A finite intra-orbital next-nearest-neighbor (NNN) hopping $t^{\prime}$, expected due to presence of Fe-As-Fe path along plaquette diagonals, is also included. Finite $t^{\prime}$ plays a very important role in stabilizing the SDW ordering.

We start with the two-orbital Hubbard model Hamiltonian:

$$
H=-\sum_{\langle i j\rangle \mu \nu \sigma} t_{i j}^{\mu \nu}\left(a_{i \mu \sigma}^{\dagger} a_{j \nu \sigma}+a_{j \nu \sigma}^{\dagger} a_{i \mu \sigma}\right)+\sum_{i \mu} U_{\mu} n_{i \mu \uparrow} n_{i \mu \downarrow}
$$

where $i, j$ refer to lattice sites; $\mu, \nu$ are the orbital indices, $t_{i j}^{\mu \nu}$ are the hopping terms as shown in Fig. 1 and $U_{\mu}$ are the intra-orbital Coulomb interactions. The role of inter-orbital density interaction and Hund's coupling will be discussed later.

In this model, the two orbitals are decoupled, with non-magnetic state dispersions $\varepsilon_{\alpha}(\mathbf{k})=-2 t \cos k_{x}-$ $4 t^{\prime} \cos k_{x} \cos k_{y}$ for the $d_{x z}$ and $\varepsilon_{\beta}(\mathbf{k})=-2 t \cos k_{y}-$ $4 t^{\prime} \cos k_{x} \cos k_{y}$ for the $d_{y z}$ orbitals. Although the dispersions are different, the energy bands are degenerate in the non-magnetic state wherein $x$ and $y$ directions are equivalent. This degeneracy is important to satisfy point-group symmetry conditions. It is noteworthy that

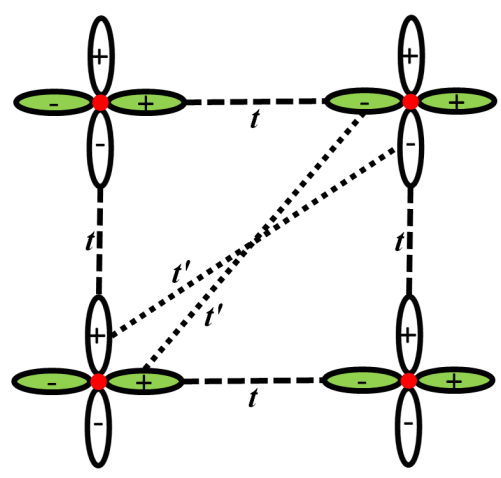

FIG. 1. Effective hopping parameters $t_{i j}^{\mu \nu}$ in the minimal twoband model involving $d_{x z}$ (filled) and $d_{y z}$ (empty) orbitals, referred to as $\alpha$ and $\beta$ respectively.

the $d_{x z}$ band along $\Gamma-Y$ and $d_{y z}$ along $\Gamma-X$ are degenerate in energy, as indeed observed in ARPES studies 13 on $\mathrm{BaFe}_{2} \mathrm{As}_{2}$ in the non-magnetic state above the ordering temperature.

In the $(\pi, 0)$ ordered SDW state, the Hartree-Fock (HF) level Hamiltonian matrix is expressed in a composite two-orbital ( $\alpha \beta$ ), two-sublattice (A B) basis as:

$H_{\mathrm{HF}}^{\sigma}(\mathbf{k})=\left[\begin{array}{cccc}-\sigma \Delta_{\alpha} & \varepsilon_{\mathbf{k}}^{x}+\varepsilon_{\mathbf{k}}^{x y} & 0 & 0 \\ \varepsilon_{\mathbf{k}}^{x}+\varepsilon_{\mathbf{k}}^{x y} & \sigma \Delta_{\alpha} & 0 & 0 \\ 0 & 0 & -\sigma \Delta_{\beta}+\varepsilon_{\mathbf{k}}^{y} & \varepsilon_{\mathbf{k}}^{x y} \\ 0 & 0 & \varepsilon_{\mathbf{k}}^{x y} & \sigma \Delta_{\beta}+\varepsilon_{\mathbf{k}}^{y}\end{array}\right]$

where $\varepsilon_{\mathbf{k}}^{x(y)}=-2 t \cos k_{x(y)}, \quad \varepsilon_{\mathbf{k}}^{x y}=-4 t^{\prime} \cos k_{x} \cos k_{y}, \quad \sigma= \pm$ for the two spins. The self-consistent exchange fields are given by $2 \Delta_{\mu}=U_{\mu} m_{\mu}$ in terms of the sublattice magnetizations $m_{\mu}$ for the two orbitals $\mu=\alpha, \beta$. As the intermediate-coupling regime will be considered throughout, the term "SDW state" is used here without any implicit weak-coupling connotation.

The density terms arising in the HF approximation, $U_{\mu} n_{\mu} / 2=\Delta_{\mu}+U_{\mu} n_{\mu}^{\downarrow}$ for the two orbitals, have not been shown explicitly in (1). In the following, we will take identical exchange fields $\Delta_{\alpha}=\Delta_{\beta} \equiv \Delta$, for which we find the relative band shift $U_{\alpha} n_{\alpha}^{\downarrow}-U_{\beta} n_{\beta}^{\downarrow}$ to be quite small, and this will be absorbed in an energy offset to be introduced later.

In iron pnictides, each iron ion has six electrons distributed among five $3 d$ orbitals. Two $e_{g}$ orbitals $d_{x^{2}-y^{2}}$ and $d_{3 z^{2}-r^{2}}$ are completely occupied by four electrons due to large crystal-field splitting between $e_{g}$ and $t_{2 g}$ states 28 , and the three $t_{2 g}$ orbitals are partially filled by the remaining two electrons. ARPES experiments 13 show that the $d_{x y}$ orbital has a finite contribution to the Fermi Surface. Therefore, the expected electron filling in our two band model will be less than half filling, and will correspond to a "hole doped" condition.

The partial densities of states for the two orbitals are shown in Fig. 2 for the non-magnetic $(\Delta=0)$ and magnetic $(\Delta /|t|=2)$ cases. While the two orbitals are de- 


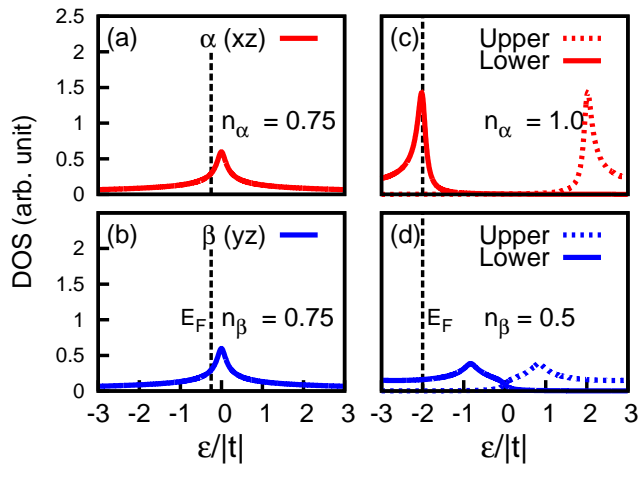

FIG. 2. Calculated partial densities of state (DOS) for $\alpha$ and $\beta$ orbitals in the non-magnetic [panels (a),(b)] and magnetic [panels (c),(d)] states. Here, $t^{\prime} / t=0.5$. The degeneracy between $\alpha$ and $\beta$ orbitals is lifted by magnetic ordering. For a typical $E_{F}$ as shown, $n_{\alpha}>n_{\beta}$. Enhanced $n_{\alpha}$ and reduced $n_{\beta}$ due to hopping anisotropy result in stronger $\mathrm{AF}$ and $\mathrm{F}$ spin couplings.

generate in the non-magnetic state, the degeneracy is lifted in the magnetic state since $x$ and $y$ directions are no longer equivalent. The hopping anisotropy together with magnetic ordering anisotropy naturally results in self orbital order in the magnetic state. For hole doping $\left(E_{F}<0\right)$, the lower $\beta$ band becomes partially unoccupied whereas the lower $\alpha$ band remains nearly half-filled. For total electron occupation $n \approx 0.75$ per orbital (i.e. $25 \%$ hole doping), we have $n_{\alpha} \approx 1.0$ and $n_{\beta} \approx 0.5$. This sign of ferro-orbital order $\left(n_{\alpha}-n_{\beta}>0\right)$ is in agreement with experiments $2-15$. The higher value of $\alpha$ DOS than $\beta$ DOS at Fermi energy naturally leads to more conductivity along $\mathrm{AF}$ direction than $\mathrm{F}$ direction which agrees with experiments 21 .

\section{TRANSVERSE SPIN FLUCTUATIONS}

Spin-wave excitations in this spontaneously-brokensymmetry SDW state are obtained from the transverse spin fluctuation propagator:

$$
\left[\chi_{\mathrm{RPA}}^{-+}(\mathbf{q}, \omega)\right]=\frac{\left[\chi^{0}(\mathbf{q}, \omega)\right]}{\mathbf{1}-[U]\left[\chi^{0}(\mathbf{q}, \omega)\right]}
$$

at the RPA level. The interaction matrix $[U]$ includes $U_{\alpha}$ and $U_{\beta}$ as diagonal matrix elements. The bare particlehole propagator $\left[\chi^{0}(\mathbf{q}, \omega)\right]$ is evaluated in the composite orbital-sublattice basis ${ }^{24}$ by integrating out the fermions in the $(\pi, 0)$ ordered SDW state as:

$$
\begin{array}{r}
{\left[\chi^{0}(\mathbf{q}, \omega)\right]_{a b}=i \int \frac{d \omega^{\prime}}{2 \pi} \sum_{\mathbf{k}^{\prime}}\left[G_{\mathrm{HF}}^{\uparrow}\left(\mathbf{k}^{\prime}, \omega^{\prime}\right)\right]_{a b}} \\
{\left[G_{\mathrm{HF}}^{\downarrow}\left(\mathbf{k}^{\prime}-\mathbf{q}, \omega^{\prime}-\omega\right)\right]_{b a}}
\end{array}
$$

where $\left[G_{\mathrm{HF}}^{\sigma}(\mathbf{k}, \omega)\right]=\left[\omega \mathbf{1}-H_{\mathrm{HF}}^{\sigma}(\mathbf{k})\right]^{-1}$ are the HF level Green's function in the SDW state and $a, b$ being the

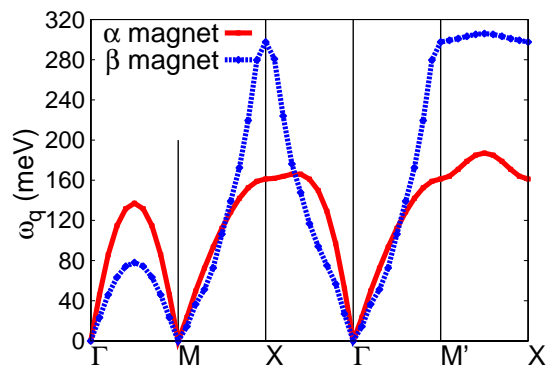

FIG. 3. Spin-wave dispersions for the two magnet modes $\alpha$ and $\beta$ along symmetry directions of the BZ for $25 \%$ hole doping. Here $t=-200 \mathrm{meV}, t^{\prime} / t=0.5, \Delta /|t|=2.0$, and the Fermi energy $E_{\mathrm{F}} /|t| \approx-2.0$.

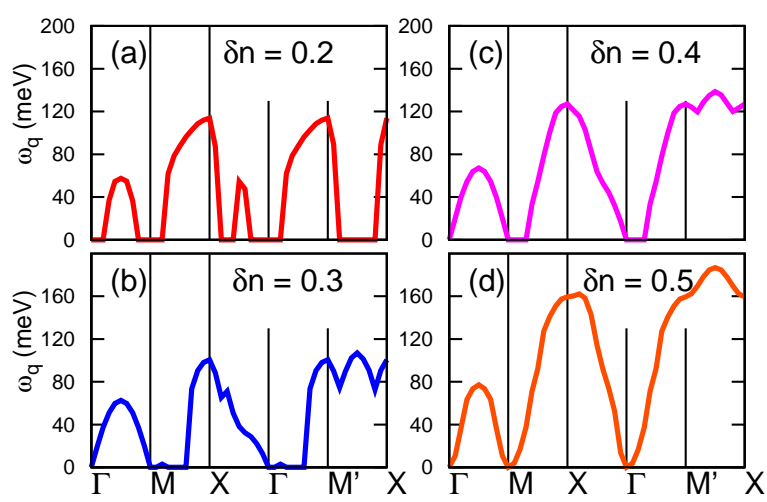

FIG. 4. Spin-wave energies with different orbital polarization $\delta n=n_{\alpha}-n_{\beta}$ for $25 \%$ hole doping. Crossover from negative to positive energy modes shows strong stabilization of the AF-F state with increasing orbital order. The hopping parameters and exchange fields are same as in Fig. 3.

orbital-sublattice basis indices. The spin-wave energies are obtained from the poles of Eq. (3).

In the absence of any Hund's coupling, the two orbitals are decoupled and the magnetic system reduces to two independent magnetic sub-systems $\alpha$ and $\beta$ involving AF ordering in $x$ direction ( $\alpha$ magnet) and $\mathrm{F}$ ordering in $y$ direction ( $\beta$ magnet). The spin-wave dispersions for the two magnet modes $\alpha$ and $\beta$ are shown in Fig. 3 along $(0,0) \longrightarrow(\pi, 0) \longrightarrow(\pi, \pi) \longrightarrow(0, \pi) \longrightarrow(\pi, \pi)[\Gamma \longrightarrow M \longrightarrow$ $\left.X \longrightarrow M^{\prime} \longrightarrow X\right]$. For $t^{\prime}=0$, the system further reduces to independent $\mathrm{AF}$ and $\mathrm{F}$ chains, which yield zero spin wave energy for wave vector in the respectively perpendicular directions (due to absence of any spin coupling along those directions), implying instabilities of the magnetic state. Fig. 3 thus highlights the important role of finite $t^{\prime}$ and the corresponding finite inter-chain spin couplings in stabilizing the two $\alpha$ and $\beta$ magnets with respect to spin twisting in any direction, as indicated by positive spin wave energies over the entire BZ.

To investigate the effect of orbital order on the SDW 
state stability, we plot the spin-wave dispersions for different orbital polarization $\delta n=n_{\alpha}-n_{\beta}$ in Fig. 4. As magnetic state instability is indicated by the spin-wave energy crossing zero and going negative, we will focus only on the lowest (out of the two $\alpha$ and $\beta$ mode) energies. Here, we have maintained a constant SDW order parameter $\Delta$ and total electron filling $\mathrm{n}=0.75$ per orbital, and the occupations $n_{\alpha}$ and $n_{\beta}$ of the two orbitals are controlled by introducing an energy offset $\Delta_{\alpha \beta}$ between the two orbitals. The orbital occupations are (a) $n_{\alpha}=0.85, n_{\beta}=0.65\left(\Delta_{\alpha \beta}=0.38\right)$, (b) $n_{\alpha}=0.9, n_{\beta}=$ $0.6\left(\Delta_{\alpha \beta}=0.29\right)$, (c) $n_{\alpha}=0.95, n_{\beta}=0.55\left(\Delta_{\alpha \beta}=0.20\right)$ and (d) $n_{\alpha}=1.0, n_{\beta}=0.5\left(\Delta_{\alpha \beta}=0\right)$. Figure 4 shows a strong stabilization of the SDW state with orbital order, as seen by crossover from negative to positive energy modes.

The origin of this orbital-order-induced stabilization is as follows. Effectively, the AF and F spin couplings are optimized by the electron density redistribution associated with orbital order. The increased electron density in $\alpha$ band favors AF coupling (super exchange) in the $x$ direction (NN) and in the diagonal direction $(\mathrm{NNN})$, whereas the increased hole density in $\beta$ band favors carrier-mediated $\mathrm{F}$ coupling in the $y$ direction $(\mathrm{NN})$ and AF coupling in the diagonal direction $(\mathrm{NNN})^{29}$. Thus, all the spin couplings work together and the $\alpha$ and $\beta$ magnets both reinforce $(\pi, 0)$ ordering without any frustration. It is important to note here that the magnetic and orbital orderings effectively stabilize each other and constitute a composite spin-orbital ordered state with $m \neq 0, \delta n \neq 0$. Our model thus provides a microscopic understanding of the close relation between in-plane anisotropy, orbital order, and the SDW magnetic order. If the inter-orbital Coulomb interaction term $V n_{i \alpha} n_{i \beta}$ is included in our model, it will only enhance the orbital offset $\Delta_{\alpha \beta}$ due to orbital disparity, and therefore enhance the effect discussed above.

Ferro orbital order was reported in a recent study of magnetic excitations in iron pnictides within a degenerate double-exchange model including antiferromagnetic superexchange interactions 27 . However, the sign of the ferro orbital order reported in this work $\left(n_{y z}>n_{x z}\right)$ does not agree with experiments. Furthermore, for a realistic NN hopping value of $200 \mathrm{meV}$, their calculated spin wave energy scale of around $30 \mathrm{meV}$ is well below the nearly $200 \mathrm{meV}$ energy scale measured in INS experiments. Ferro orbital was also reported in other multiorbital models due to electron correlation ${ }^{31}$, anisotropic inter-orbital hopping 32 and electron-lattice coupling 33 . However, spin wave excitations and role of orbital ordering in stabilization of $(\pi, 0)$ state with respect to transverse spin fluctuations was not investigated. Moreover, crystal field splitting due to orthorhombic distortion is necessary to stabilize orbital ordering in these models unlike our model.

Now, we investigate the effect of finite inter-orbital

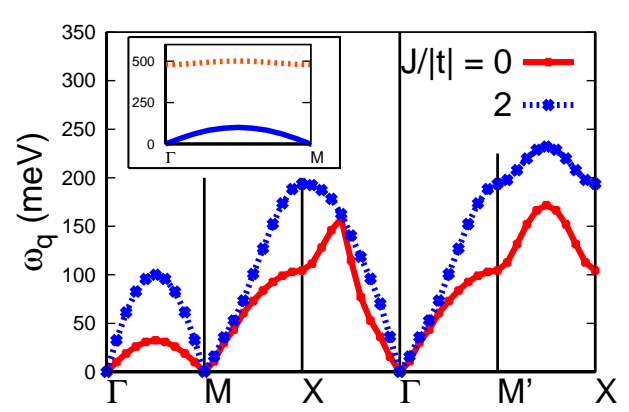

FIG. 5. Strong enhancement in the spin-wave energies on including the Hund's coupling. Here, $t=-200 \mathrm{meV}, t^{\prime} / t=0.3$, $\Delta /|t|=2.0$, and hole doping $25 \%$.

Hund's coupling $J$, and consider the Hamiltonian:

$$
H=-\sum_{\langle i j\rangle \mu \nu \sigma} t_{i j}^{\mu \nu}\left(a_{i \mu \sigma}^{\dagger} a_{j \nu \sigma}+a_{j \nu \sigma}^{\dagger} a_{i \mu \sigma}\right)-\sum_{i \mu \nu} U_{\mu \nu} \mathbf{S}_{i \mu} \cdot \mathbf{S}_{i \nu}
$$

where the interaction matrix elements $U_{\mu \nu}=U_{\mu}$ for $\mu=\nu$ and $U_{\mu \nu}=2 J$ for $\mu \neq \nu$. The self-consistent exchange fields are now given by $2 \Delta_{\alpha}=U_{\alpha} m_{\alpha}+J m_{\beta}$ and $2 \Delta_{\beta}=U_{\beta} m_{\beta}+$ $J m_{\alpha}$.

The transverse spin fluctuation propagator now includes both $U$ and $J$ ladders at RPA level. Figure 5 shows the spin-wave dispersion with and without Hund's coupling, displaying a strong enhancement of spin-wave energies with Hund's coupling, as expected since now the $\alpha$ and $\beta$ magnets are coupled.

For $J=0$, there are two independent Goldstone modes, as the $\alpha$ and $\beta$ magnets are independent. When finite Hund's coupling is included, the two modes get coupled, leading to a single Goldstone mode corresponding to "inphase" fluctuations (acoustic mode). The "out-of-phase" mode is now converted to an optical branch which rapidly becomes significantly gapped even at small values of $J$. The spin-waves for the acoustic and optical modes (solid and dotted respectively) are shown in the inset [Fig. 5].

The calculated spin-wave energy scale $(\sim 200 \mathrm{meV})$ agrees well with neutron scattering experiments for $|t|=200 \mathrm{meV}$ and $U$ in the intermediate coupling range $(U \sim W)$. The value of $\mathrm{U}(\sim 1-2 \mathrm{eV})$ agrees with LDA calculations ${ }^{30}$. Furthermore, the spin-wave dispersion shows a peak at the $\mathrm{F}$ zone boundary which is consistent with experiments. For $\Delta /|t|=2.0$, the SDW state effective gap $\sim 400 \mathrm{meV}$ is well above the maximum spin wave energy. Thus, contrary to other itinerant models 9 , spin wave excitations in our model do not rapidly dissolve into the particle-hole continuum, as indeed not observed experimentally up to energies of $200 \mathrm{meV}$.

The spin wave energy scales in the $\Gamma-\mathrm{M}(\mathrm{AF})$ and $\mathrm{M}-$ $\mathrm{X}(\mathrm{F})$ directions are indicative of the $\mathrm{AF}$ and $\mathrm{F}$ spin coupling strengths. The hole doping dependence of spin wave dispersion (Fig. 6) effectively shows the evolution of emergent spin couplings. At high hole doping, the 


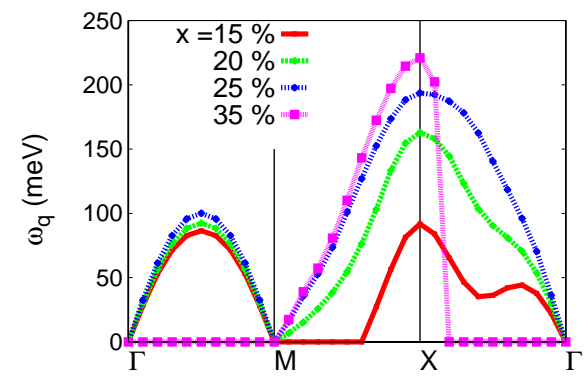

FIG. 6. Variation of spin wave dispersion with hole doping, showing strong suppression of AF (F) spin couplings at high (low) doping. Here $t=-200 \mathrm{meV}, t^{\prime} / t=0.3$, and $\Delta=J=2|t|$.

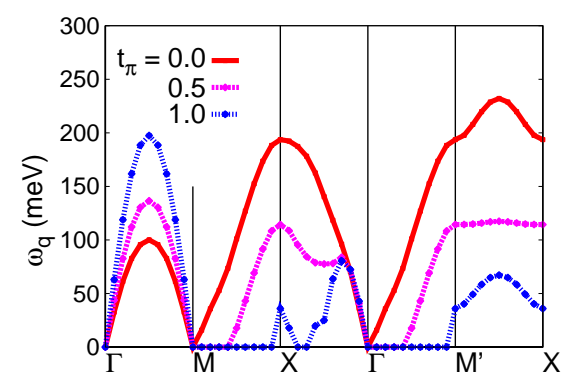

FIG. 7. Strong enhancement in spin wave energy scale and SDW state stability with increasing hopping anisotropy. Here $t=-200 \mathrm{meV}, t^{\prime} / t=0.3, \Delta=J=2|t|$, and hole doping $25 \%$.

AF spin coupling is strongly suppressed due to electron density depletion in the AF band, whereas the $\mathrm{F}$ spin coupling is optimized due to enhanced hole doping in the $\mathrm{F}$ band. On the other hand, at low hole doping, the AF spin coupling gets saturated as the AF (lower) band is filled, whereas the F spin coupling is strongly weakened.

Maximum SDW state stability is seen for about $25 \%$ hole doping. If this corresponds to the parent compound, then electron doping (reduction in hole doping from this level) results in crossover to negative-energy modes, indicating destabilization of the SDW state. This is in agreement with the observed rapid decrease of magnetic ordering temperature in iron pnictides with electron doping $\stackrel{\mathbb{1}}{ }$.

Spin waves were studied earlier ${ }^{24}$ in a two-band model with isotropic hopping and therefore no orbital order. For somewhat larger interaction strength $(\Delta /|t|)=3)$ and hole doping $(\sim 40 \%)$, the spin wave energies are qualitatively similar as in Fig. 5. However, for lower interaction strength and hole doping, increasing hopping anisotropy significantly enhances the spin wave energy scale and SDW state stability, as shown in Fig. 7 This confirms that the emergent AF and $\mathrm{F}$ spin couplings become stronger due to the electron density redistribution and orbital order in the two orbitals, corresponding to enhanced electron and hole densities in the $d_{x z}$ and $d_{y z}$ Fe orbitals, respectively.

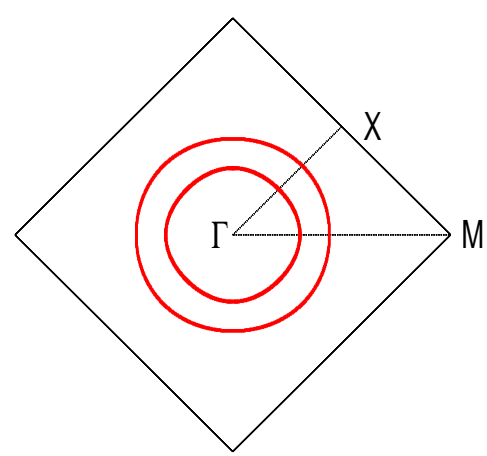

FIG. 8. Fermi surface in the folded BZ for $t_{1}=-1.0, t_{2}=0.5$, $t_{3}=t_{4}=-0.5$, where the hopping parameters are defined in the same way as in 23 . Here, $E_{F}=-0.3\left|t_{1}\right|$ for $25 \%$ hole doping.

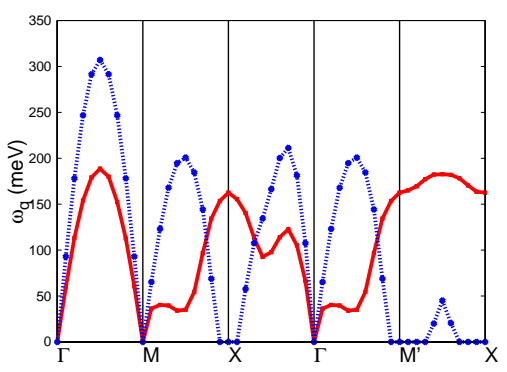

FIG. 9. Spin wave dispersion for the same parameters as in Fig. 8 (solid line) and for nesting condition as in ${ }^{23}$ (dotted line). Here, $\Delta=3.0\left|t_{1}\right|, J=2.0\left|t_{1}\right|$ and $\left|t_{1}\right|=200 \mathrm{meV}$. The finite spin wave energy at $\mathrm{M}$ ' indicates emergent $\mathrm{F}$ spin coupling.

\section{MODIFIED VERSION OF TWO-BAND MODEL OF RAGHU ET AL.}

With the insight thus obtained, we consider a modified version of the two-band model of Raghu et al. which retains most of their essential features, and simultaneously yields not only the two circular hole pockets, but also appropriate orbital order, and also evidence of emergent F spin couplings. The hopping parameters are defined in the same way as $i^{23} \underline{3}$ and their values are taken as $t_{1}=-1.0, t_{2}=0.5, t_{3}=t_{4}=-0.5$. The corresponding Fermi surface and the spin wave dispersion for $25 \%$ hole doping are shown in Fig. 8 and Fig. 9, respectively. The finite spin wave energy at the F zone boundary M' implies the emergence of $\mathrm{F}$ spin coupling. Although nesting is no longer obtained due to absence of electron pockets, we have $n_{\alpha} \simeq n_{\beta} \simeq 0.75$ in paramagnetic state, $n_{\alpha} \simeq 0.82, n_{\beta} \simeq 0.68$ in $(\pi, 0)$ state and hence orbital order of correct sign is obtained in this model. This suggests that an additional $d_{x y}$ band may need to be included to simultaneously understand all the important electronic Fermi surface features as observed in ARPES experiments (including the elliptical electron pockets) 
and the spin wave features as observed in INS studies.

\section{CONCLUSION}

In conclusion, we have shown that within a minimal two-orbital itinerant-electron model with hopping anisotropy, the magnetically anisotropic $(\pi, 0)$ ordered SDW state is stabilized through the generation of orbital order which optimizes both AF and F spin couplings. We have also shown that with the inclusion of inter-orbital Hund's coupling, the spin stiffness further increases substantially due to the coupling of the two magnetic subsystems. The calculated spin-wave energy scale, nature of spin-wave dispersion and presence of ferro-orbital order are in agreement with experimental observations. Thus, the proclivity of iron arsenides towards $(\pi, 0)$ magnetic ordering may actively involve the orbital degree of freedom. We have also considered a two-band model taking hopping parameters similar to Raghu et al $\stackrel{23}{n}$, but with hopping anisotropy, which yields appropriate orbital order, circular hole pockets, as well as emergent $\mathrm{F}$ spin couplings. The presence of orbitally ordered state in our model allows the possibility of exploring orbital fluctuations which have been seen in recent experiments 15 . Orbital fluctuations may play an important role on the electron-paring mechanism ${ }^{34}$ and can induce $s++$ wave superconducting state ${ }^{35}$, and therefore a combination of orbital and spin fluctuations as a possible mechanism for superconductivity also needs to be explored in these materials.

Sayandip Ghosh acknowledges financial support from Council of Scientific and Industrial Research, India.

\section{REFERENCES}

${ }^{1}$ J. Zhao, Q. Huang, C. de la Cruz, S. Li, J. W. Lynn, Y. Chen, M. A. Green, G. F. Chen, G. Li, Z. Li, J. L. Luo, N. L. Wang, and P. Dai Nature Mat. 7, 953 (2008).

${ }^{2}$ S. Nandi, M. G. Kim, A. Kreyssig, R. M. Fernandes, D. K. Pratt, A. Thaler, N. Ni, S. L. Bud'ko, P. C. Canfield, J. Schmalian, R. J. McQueeney, and A. I. Goldman Phys. Rev. Lett. 104, 057006 (2010).

${ }^{3}$ A .I. Goldman, D. N. Argyriou, B. Ouladdiaf, T. Chatterji, A. Kreyssig, S. Nandi, N. Ni, S. L. Bud'ko, P. C. Canfield, and R. J. McQueeney Phys. Rev. B 78, 100506(R) (2008).

${ }^{4}$ J. Zhao, D. T. Adroja, D-X Yao, R. Bewley, S. Li, X. F. Wang, G. Wu, X. H. Chen, J. Hu, and P. Dai Nature Phys. 5, 555 (2009). ${ }^{5}$ S. O. Diallo, V. P. Antropov, T. G. Perring, C. Broholm, J. Pulikkotil, N. Ni, S. L. Bud'ko, P. C. Canfield, A. Kreyssig, A. I. Goldman, and R.J. McQueeny Phys. Rev. Lett. 102, 187206 (2009).

${ }^{6}$ R.A. Ewings, T. G. Perring, J. Gillett, S. D. Das, S. E. Sebastian, A. E. Taylor, T. Guidi, and A. T. Boothroyd Phys. Rev. B 83, 214519 (2011).

${ }^{7}$ I. Eremin, and A. V. Chubukov Phys. Rev. B 81, 024511 (2010).

${ }^{8}$ P. M. R. Brydon, and C. Timm Phys. Rev. B 80, 174401 (2009).
${ }^{9}$ J. Knolle, I. Eremin, A. V. Chubukov, and R. Moessner Phys. Rev. B 81 140506(R) (2010).

${ }^{10}$ K. Nakamura, R. Arita, and M. Imada J. Phys. Soc. Jpn. 77, 093711 (2008).

${ }^{11}$ S. L. Skornyakov, A. V. Efremov, N. A. Skorikov, M. A. Korotin, Y. A. Izyumov, V. I. Anisimov, A. V. Kozhevnikov, and D. Vollhardt Phys. Rev. B 80, 092501 (2009).

${ }^{12}$ T. Shimojima, K. Ishizaka, Y. Ishida, N. Katayama, K. Ohgushi, T. Kiss, M. Okawa, T. Togashi, X-Y Wang, C-T Chen, S. Watanabe, R. Kadota, T. Oguchi, A. Chainani, and S. Shin Phys. Rev. Lett. 104, 057002 (2010).

${ }^{13}$ M. Yi, D. Lu, J.-H. Chu, J. G. Analytis, A. P. Sorini, A. F. Kemper, B. Moritz, S.-K. Mo, R. G.Moore, M. Hashimoto, W.S. Lee, Z. Hussain , T. P. Devereaux, I. R. Fisher, and Z-X Shen Proc. Nat. Ac. Science 108, 6878 (2011).

${ }^{14}$ M. F. Jensen, V. Brouet, E. Papalazarou, A. Nicolaou, A. TalebIbrahimi, P. Le Fèvre, F. Bertran, A. Forget, and D. Colson D Phys. Rev. B 84014509 (2011).

${ }^{15}$ Y. K. Kim, W. S. Jung, G. R. Han, K.-Y. Choi, K.-H. Kim, C.-C. Chen, T. P. Devereaux, A. Chainani, J. Miyawaki, Y. Takata, Y. Tanaka, M. Oura, S. Shin, A. P. Singh, H. G. Lee, J.-Y. Kim, and C. Kim Phys. Rev. Lett. 111, 217001 (2013).

${ }^{16}$ W. Lv, J. Wu, and P. Phillips Phys. Rev. B 80, 224506 (2009).

${ }^{17}$ C.-C. Lee, W.-G. Yin, and W. Ku Phys. Rev. Lett. 103, 267001 (2009).

${ }^{18}$ C.-C. Chen, J. Maciejko, A. P. Sorini, B. Moritz, R. R. P. Singh, and T. P. Devereaux Phys. Rev. B 82, 100504(R) (2010).

${ }^{19}$ W. Lv, and P. Phillips Phys. Rev. B 84174512 (2011).

${ }^{20}$ M. A. Tanatar, E. C. Blomberg, A. Kreyssig, M. G. Kim, N. Ni, A. Thaler, S. L. Bud'ko, P. C. Canfield, A. I. Goldman, I. I. Mazin, and R. Prozorov Phys. Rev. B 81, 184508 (2010).

${ }^{21}$ J-H Chu, J. G. Analytis, K. De Greve, P. L. McMahon, Z. Islam, Y. Yamamoto, I. R. Fisher Science 329, 824 (2010).

${ }^{22}$ T-M Chuang, M. P. Allan, J. Lee, Xie Yang, Ni Ni, S. L. Bud'ko, G. S. Boebinger, P. C. Canfield, and J. C. Davis Science 327, 181 (2010).

${ }^{23}$ S. Raghu, X.-L. Qi, C.-X. Liu, D. J. Scalapino, and S.-C. Zhang Phys. Rev. B 77, 220503(R) (2008).

${ }^{24}$ N. Raghuvanshi, and A. Singh J. Phys.: Cond. Mat. 23, 312201 (2011).

${ }^{25}$ J. Knolle, I. Eremin , and R. Moessner Phys. Rev. B 83, 224503 (2011).

${ }^{26}$ N. Raghuvanshi, and A. Singh J. Phys.: Cond. Mat. 22, 422202 (2010).

${ }^{27}$ W. Lv, F. Krüger, and P. Phillips Phys. Rev. B 82, 045125 (2010).

${ }^{28}$ F. Krüger, S. Kumar, J. Zaanen, and J. van den Brink Phys. Rev. B 79, 054504 (2009).

${ }^{29}$ A. Singh, and H. Ghosh Phys. Rev. B 65, 134414 (2002).

${ }^{30}$ W. L. Yang, A. P. Sorini, C.-C. Chen, B. Moritz, W.-S. Lee, F. Vernay, P. Olalde-Velasco, J. D. Denlinger, B. Delley, J.-H. Chu, J. G. Analytis, I. R. Fisher, Z. A. Ren, J. Yang, W. Lu, Z. X. Zhao, J. van den Brink, Z. Hussain, Z.-X. Shen, and T. P. Devereaux Phys. Rev. B 80, 014508 (2009).

${ }^{31}$ Y.-M. Quan, L.-J. Zou, D.-Y. Liu, and H.-Q. Lin J. Phys.: Cond. Mat. 24, 085603 (2012).

${ }^{32}$ E. Bascones, M. J. Calderon, and B. Valenzuela Phys. Rev. Lett. 104, 227201 (2010).

${ }^{33}$ D.-Y. Liu, Y.-M. Quan, D.-M. Chen, L.-J. Zou, and H.-Q. Lin Phys. Rev. B 84, 064435 (2011).

${ }^{34}$ T. Shimojima, F. Sakaguchi, K. Ishizaka, Y. Ishida, T. Kiss, M. Okawa, T. Togashi, C.-T. Chen, S. Watanabe, M. Arita, K. Shimada , H. Namatame, M. Taniguchi, K. Ohgushi, S. Kasahara, T. Terashima, T. Shibauchi, Y. Matsuda, A. Chainani, and S. Shin Science 332, 564 (2011).

${ }^{35}$ T. Saito, S. Onari, and H. Kontani Phys. Rev. B 82, 144510 (2010). 